\title{
Panic disorder
}

\section{P Szabo}

\section{Introduction}

Panic disorder (PD) is a prevalent anxiety disorder with lifetime prevalence rates ranging from $1.1 \%$ to $3.7 \%$ in the general population and $3.0 \%$ to $8.3 \%$ in clinic settings. ${ }^{[1]}$ The presence of agoraphobia in patients with PD is associated with substantial severity, comorbidity (e.g. major depression, other anxiety disorders, alcohol abuse) and functional impairment. ${ }^{[1]}$ The disorder is more common in women than in men, with a 3:1 ratio in patients with agoraphobia and 2:1 in patients without agoraphobia. While panic attacks are a core feature of $\mathrm{PD}$, panic attacks are also experienced by patients with post-traumatic stress disorder, social anxiety disorder and specific phobias. However, unlike in PD, these are typically cued by exposure to or anticipation of specific anxiety-provoking situations. ${ }^{[2]}$

\section{Diagnosis and clinical characteristics}

$\mathrm{PD}$ is an anxiety disorder characterised by recurrent panic attacks involving intense fear/discomfort and accompanied by at least 4 of 13 somatic or cognitive symptoms which develop abruptly and reach a peak within 10 minutes (Diagnostic and Statistical Manual of Mental Disorders, Fourth Edition, Text Revision (DSM-IV-TR)). ${ }^{[2]}$ Attacks should not be substance-induced, nor related to a medical condition or as a consequence of another psychiatric disorder, and should be spontaneous in nature. Panic attacks that occur with fewer than 4 of the 13 panic symptoms are termed limited symptom attacks. ${ }^{[2]}$ To make the diagnosis of $\mathrm{PD}$, at least one of the attacks must be followed by a month or more of persistent concern regarding the possibility of a subsequent attack, worry about the implications of the attacks and/or behavioural change, e.g. avoidant behaviours such as agoraphobia - anxiety about being in places from which escape might be difficult or where help may not be available in the event of a panic attack. ${ }^{[2]}$ PD may occur with or without agoraphobia. ${ }^{[2]}$ The disorder has been described as a 'common, persistent and disabling' condition. ${ }^{[3]}$ Notwithstanding such a description, both pharmacological and psychotherapeutic interventions have established efficacy.

\section{Assessment}

Based on the clinical characteristics, and awareness of the potential diagnostic pitfalls, i.e. substance/medically/other related psychiatric disorders, the assessment requires not only a careful history but also the possibility of toxic screening and physical investigation to rule out medically or substance-related presentations.

\section{Treatment}

\subsection{Treatment goals}

The initial goal of any intervention is symptom relief together with maintenance of functioning, followed by ongoing alleviation of symptoms accompanied by optimal functioning. PD represents a specific challenge, given the experience of panic attacks as events characterised by fear and accompanied by a range of somatic symptoms. ${ }^{[2]}$ Therefore the goal remains to reduce the severity and intensity of panic attacks, avoidance, fearful anticipation, and cognitive distortions. Of specific relevance is the unpredictability of episodes and the need for clinicians to meaningfully reassure patients of the planned intervention in terms of outcome, for both future episodes and functioning.

\subsection{General aspects of treatment}

Two broad categories of intervention have demonstrable efficacy, i.e. pharmacological and psychotherapeutic. Both interventions may serve as first-line treatments, as meta-analytic reviews and large-scale comparative trials have shown comparable efficacy, with high remission rates $(60-80 \%)$ and maintenance of gains over time for both modalities. ${ }^{[4]}$ Systematic reviews have also confirmed that a combination of the two is most effective in the acute phase with ongoing superior effectiveness following the acute-phase treatment. This is compared to pharmacotherapy alone; however, combination therapy may offer only limited benefits beyond that derived from psychotherapy alone (viz. cognitive-behavioural therapy (CBT)). Pharmacotherapy includes the use of both antidepressant agents and benzodiazepines. Psychotherapeutic approaches include both cognitive and behavioural components either individually or in combination. ${ }^{[5]}$

\subsection{Pharmacological treatment: Acute}

The first-line pharmacotherapy of choice in this anxiety disorder is the selective serotonin reuptake inhibitors (SSRIs, e.g. fluoxetine/ paroxetine/fluvoxamine/sertraline/citalopram/escitalopram), or the serotonin-norepinephrine reuptake inhibitor (SNRI) venlafaxine (including the extended-release formulation), with multiple randomised controlled trials (RCTs) showing their efficacy and safety. ${ }^{[3]} \mathrm{PD}$ also responds to certain other antidepressants, such as the tricyclic antidepressants (TCAs) (while of equivalent efficacy to SSRIs, their use is limited by side-effects). ${ }^{[3]}$ Very-low-dose treatment should be initiated in PD (e.g. fluoxetine $5 \mathrm{mg}$ /venlafaxine ER 75 $\mathrm{mg}$ ) with gradual upward titration as required. At least $6-8$ weeks of adequate doses of medication are required to assess whether an acute intervention is effective or not. While such an approach appears reasonable, the use of antidepressants as stand-alone, first-line intervention has, on the basis of systematic review, been cautioned against. ${ }^{[4]}$ Although there is less evidence available for children/ adolescents, SSRIs may again be useful. ${ }^{[6,7]}$

Given concerns about their side-effect profile (as well as tolerance and dependency), the use of high-potency benzodiazepines should generally be limited to short-term augmentation of antidepressant medication to rapidly stabilise PD symptoms. ${ }^{[8]}$ Certainly the use of combination treatment (clonazepam/sertraline) has been found to provide rapid - and safe - stabilisation of panic symptoms. ${ }^{[9]}$ Benzodiazepines (alprazolam, clonazepam and lorazepam) are effective 
and use patterns have demonstrated that this class of drug is the most commonly used, notwithstanding guidelines recommending SSRIs as the preferred treatment. ${ }^{[10]}$ Data regarding the use of benzodiazepine treatment combined with psychotherapy versus benzodiazepine treatment alone suggest superiority of combined treatment; however, there is a paucity of good-quality evidence, either supportive or nonsupportive. ${ }^{[11]}$ Of note is a systematic review that failed to identify quality studies comparing benzodiazepines to newer antidepressants (now regarded as the first line of pharmacological intervention), with the suggestion that the promoted move away from first-line and maintenance treatment with benzodiazepines has occurred without appropriate evidence to support this approach. ${ }^{[12]}$ Most typically the benzodiazepines are seen to have value as 'rescue' agents, specifically alprazolam (including the extended-release formulation), ${ }^{[13]}$ in spite of a lack of evidence-based data supporting such use, notwithstanding clinician and patient support and preference for such use. ${ }^{[14]}$

\subsection{Pharmacological treatment: Maintenance}

Maintenance treatment at the same dose on which improvement occurred should be continued for at least 1 year. Relapse rates following discontinuation have shown varying outcomes, with rates ranging from $25 \%$ to $50 \%{ }^{[2]}$ Of particular interest is that ongoing psychotherapy (CBT) is as effective as combination treatment (pharmacotherapy/psychotherapy), which suggests that this may be the preferred option in the longer term.

\subsection{Non-pharmacological treatment}

CBT has been shown to be effective for the treatment of anxiety disorders in multiple RCTs. ${ }^{[3]} \mathrm{CBT}$ for PD consists of components of psycho-education, cognitive restructuring, and behavioural interventions, with the combination of exposure therapy, relaxation exercises and breathing retraining providing the most consistent evidence for efficacy. ${ }^{[4]}$ The typical duration of treatment is $12-15$ sessions, but even briefer treatments have shown efficacy. ${ }^{[4]}$ The combination of pharmacotherapy and psychotherapy may have particular advantages (e.g. preventing relapse after medication discontinuation) and has been suggested as a first line of treatment (together with psychotherapy alone). ${ }^{[5]}$ In addition to these more traditional non-pharmacological interventions, the role of education, self-management and internet-based interventions have shown benefit and appear worthy of further study for select patients and situations. ${ }^{[3]}$ Also, the use of virtual-reality exposure therapy represents an interesting development, although it has not been clearly demonstrated to be superior to CBT. ${ }^{[3]}$

\subsection{Special populations}

Comorbidity is common among psychiatric populations. Within the context of PD, the impact of panic symptoms in relation to outcomes of mood disorders is of relevance, because persistent panic symptoms appear to negatively influence treatment outcomes of major depression. ${ }^{[15]}$ Further, PD comorbid with bipolar disorder confers an increased risk for suicide. ${ }^{[16]}$ This underscores data demonstrating that PD is independently associated with risk for suicide attempts. ${ }^{[16]}$ The treatment of PD among patients with comorbid bipolar disorder represents a challenge in so far as the use of antidepressant agents may induce mania. ${ }^{[17]}$ Further, the presence of mood instability in PD patients may worsen the condition, as well as lead to resistance to antidepressants. ${ }^{[18]} \mathrm{A}$ recently published trial of sodium valproate, at doses of $600-700 \mathrm{mg}$ daily (commencing at $300 \mathrm{mg}$ daily), co-administered with an antidepressant or as monotherapy in PD patients with comorbid bipolar disorder, produced symptom remission. ${ }^{[18]}$ There are limited controlled data in this regard; hence Perugi et al.$^{[18]}$ provide limited but objective evidence to support the use of mood stabilisers as treatment for PD, in both 'antidepressantresistant' sufferers as well as those with comorbid bipolar disorder. Another population of interest are those suffering from migraine where PD is strongly associated, with either condition impacting on the other bidirectionally. ${ }^{[19]}$ It is clear that clinical assessment regarding comorbidity covers a range of conditions and is critical for outcome and optimal treatment of PD. Of specific interest are those patients with anxiety disorders, including $\mathrm{PD}$, who have comorbid substance-related conditions, specifically in relation to benzodiazepine use. It appears that the evidence does not preclude the use of such agents in these patients. ${ }^{[20]}$

\subsection{Partial and non-responders}

Reviewing the studies for pharmacological management strategies, the majority of patients treated with a range of antidepressant agents achieve panic-free status during a trial of medication (with no clear cut dose-response relationship) with generally high rates of response and somewhat lower rates of remission. ${ }^{[3]}$ It should be noted that placebo response rates of up to $50 \%$ also occur, ${ }^{[3]}$ and when existing data have been subjected to systematic review, combination treatment (pharmacotherapy plus psychotherapy) response rates of just over $50 \%$ have been found. ${ }^{[5]}$ Most patients do respond, to varying degrees, to active treatment with a range of interventions that include both pharmacotherapy and psychotherapy, either alone or in combination. Obviously when either partial or non-response is encountered, accurate diagnosis is paramount; careful reassessment should occur. Multiple agents, at optimal doses for adequate duration as well as combination treatment, are all considerations when confronted with partial or non-response - specifically combinations of antidepressant medication and benzodiazepines. It has been suggested that benzodiazepine use be reserved for treatment-resistant patients, ${ }^{[15,21]}$ but it appears that actual clinical practice sees the use of benzodiazepines far more routinely and in various ways. ${ }^{[22]}$ While there are limited data related to treatment resistance, the role of mood instability has been postulated as a factor contributing to 'resistance to antidepressants' with a study of sodium valproate demonstrating improved outcomes where used as an adjunct or as monotherapy (doses ranging from 300 to $700 \mathrm{mg}$ daily). ${ }^{[18]}$ Various other agents, i.e. gabapentin, olanzapine and quetiapine, have demonstrated efficacy in the treatment of PD and could potentially be considered under circumstances of partial or non-response. ${ }^{[17]}$ Within this context there has been increasing interest in the use of natural remedies for the treatment of anxiety disorders, of which inositol has been studied with regard to PD. ${ }^{[23]}$ In the most recent of such studies inositol (18 g/ day) was compared to fluvoxamine ( $150 \mathrm{mg} /$ day) using a double-blind crossover approach which demonstrated equivalent overall efficacy in the treatment of PD with inositol demonstrating superiority with 
regard to reduction in the number of panic attacks. ${ }^{[24]}$ Such a study would need to be replicated before a firm recommendation could be made. In addition, the use of cognitive enhancers in the form of D-cycloserine (a partial agonist at the N-methyl-d-aspartate receptor) in conjunction with exposure therapy has shown promise but this option cannot be recommended given the paucity of data. ${ }^{[3]}$

\section{Conclusion}

Based on the existing data, it would appear that combination treatment (pharmacotherapy plus psychotherapy) is the intervention of choice, and that the judicious use of benzodiazepines together with an antidepressant will provide the most rapid initial response. Further combination treatment may ultimately be adequate in the longer term for maintenance of clinical improvement. However, maintenance should include the consideration that psychotherapy alone may be an option following longer term treatment and stabilisation on combination treatment. The data for partial or non-responders are limited, but there is a suggestion that sodium valproate may be a consideration either as an adjunctive agent or as monotherapy, specifically if there is mood instability or comorbid bipolar disorder.

\section{Summary points}

- Both pharmacological therapies and CBT are considered first-line treatments for PD.

- Antidepressant agents have proven efficacy in the treatment of PD.

- Agents from within the SSRIs and SNRIs are the preferred options, namely the SSRIs citalopram, escitalopram, fluvoxamine, fluoxetine, paroxetine, sertraline and the SNRI venlafaxine.

- Adjunctive benzodiazepines have a role in treatment.

- For acute treatment, a combination of pharmacological treatment and CBT is likely to be more effective than either therapy alone.

- CBT and pharmacotherapy are not necessarily added concurrently - there is some evidence that adding CBT to patients previously treated with pharmacotherapy provides good benefits.

- Treatment with psychotherapy alone may be the preferred longerterm option.

- Acute treatment: Combination treatment (pharmacotherapy + psychotherapy or pharmacotherapy + pharmacotherapy (e.g. antidepressant plus benzodiazepine/sodium valproate).

- Maintenance treatment: Combination treatment or psychotherapy alone.

- In children and adolescents with PD, there are only nonrandomised, controlled studies to support the utility of the SSRIs.

- CBT is a good alternative for women with PD who plan to become pregnant, and for pregnant women who need to discontinue medication.

\section{References}

1. Kessler RC, Chiu WT, Jin R, et al. The epidemiology of panic attacks, panic disorder and agoraphobia in the National Comorbidity Survey Replication. Arch Gen Psychiatry 2006;63:415-24. [http://dx.doi.org/10.1001/archpsyc.63.4.415]

2. American Psychiatric Association. Diagnostic and Statistical Manual of Mental Disorders, Fourth Edition, Text Revision (DSM-IV-TR). Washington, DC: American Psychiatric Association, 2000.

3. Pull CB, Damsa C. Pharmacotherapy of panic disorder. Neuropsychiatric Disease and Treatment 2008;4:779-795.

4. McHugh RK, Smits JA, Otto MW. Empirically supported treatments for panic disorder Psychiatr Clin North Am 2009;32:593-610. [http//:dx.doi.org/10.1016/j.psc.2009.05.005.]

5. Furukawa TA, Watanabe N, Churchill R. Psychotherapy plus antidepressant for panic disorder with or without agoraphobia. Br J Psychiatry 2006;188:305-312. [http://dx.doi.org/10.1192/ bjp.188.4.305]

6. American Academy of Child and Adolescent Psychiatry. Practice parameters for the assessment and treatment of children and adolescents with posttraumatic stress disorder. J Am Acad Child Adolesc Psychiatry 1998;37S:4-26.

7. Hawkridge S, Stein DJ. Risk-benefit assessment of drug therapies for anxiety disorders in children and adolescents. Drug Safety 1998;19:283-297.

8. Ballenger JC, Davidson JRT, Lecrubier Y, et al. Consensus statement on panic disorder from the International Consensus Group on Depression and Anxiety. J Clin Psychiatry 1998;59:47 54.

9. Goddard AW, Brouette T, Almai A, et al. Early coadministration of clonazepam with sertraline for panic disorder. Arch Gen Psychiatry 2001;58:681-686. [http://dx.doi.org/10.1001/ archpsyc.58.7.681]

10. Bruce SE, Vasile RG, Goisman RM, et al. Are benzodiazepines still the medication of choice for patients with panic disorder with or without agoraphobia? Am J Psychiatry 2003;160:14321438 .

11. Watanabe N, Churchill R, Furukawa TA. Combination of psychotherapy and benzodiazepines versus either therapy alone for panic disorder: a systematic review. BMC Psychiatry 2007;7:18.

12. Berney P, Halperin D, Tango R, et al. A major change of prescribing pattern in absence of adequate evidence: benzodiazepines versus newer antidepressants in anxiety disorders. Psychopharmacology Bulletin 2008;41:39-47.

13. Sheehan DV, Sheehan KH, Raj BA. The speed of onset of action of alprazolam-XR compared to alprazolam-CT in panic disorder. Psychopharmacology Bulletin 2007;40;63-81.

14. Westra HA, Stewart SH. As-needed use of benzodiazepines in managing clinical anxiety: incidence and Implications. Current Pharmaceutical Design 2002;8:59-74.

15. 15. DeVeaugh-Geiss AM, West SL, Miller WC, et al. Depression and comorbid panic in primary care patients. J Affect Disord 2010;123:283-290. [http://dx.doi.org/10.1016/j. jad.2009.09.013]

16. 16. Kilbane EJ, Gokbayrak NS, Galykner I, et al. A review of panic and suicide in bipolar disorder: does comorbidity increase risk? J Affect Disord 2009;115:1-10. [http://dx.doi. org/10.1016/j.jad.2008.09.014]

17. 17. El-Mallakh RS, Hollifield M. Comorbid anxiety in bipolar disorder alters treatment and prognosis. Psychiatr Q 2008;79:139-150. [http://dx.doi.org/10.1007/s11126-008-9071-5]

18. 18. Perugi G, Frare F, Toni C, et al. Adjunctive valproate in panic disorder patients with comorbid bipolar disorder or otherwise resistant to standard antidepressants: a 3 year 'open’ follow-up study. Eur Arch Psychiatry ClinNeurosci 2010;260:553-560. [http://dx.doi. org/10.1007/s00406-010-0109-y]

19. 19. Nepon J, Belik SL, Bolton J, Sareen J. The relationship between anxiety disorders and suicide attempts: findings from the national epidemiological survey on alcohol and related conditions. Depress Anxiety 2010;27:791-798. [http://dx.doi.org/10.1002/da.20674]

20. 20. Beghi E, Bussone G, D'Amico D, et al. Headache, anxiety and depressive disorders: the HADAS study. Journal of Headache and Pain 2010;11:141-150.

21. 21. Lader M. Management of panic disorder. Expert Review of Neurotherapeutics 2005;5:259266. [http://dx.doi.org/10.1007/s10194-010-0187-2]

22. 22. Posternak MA, Mueller TI. Assessing the risks and benefits of benzodiazepines for anxiety disorders in patients with a history of substance abuse or dependence. Am J Addict 2001;10:48-68.

23. 23. Kinrys G, Coleman E, Rothstein E. Natural remedies for anxiety disorders: potential use and clinical applications. Depress Anxiety 2009;26:259-265.

24. [http://dx.doi.org/10.1002/da.20460]

25. 24. Palatnik A, Frolov K, Fux M, Benjamin J. Double-blind, controlled, crossover trial of inositol versus fluvoxamine for the treatment of panic disorder. J Clin Psychopharmacol 2001;21:335-339. 